\title{
EPIDEMIOLOGISCHES PROFIL VON UNFÄLLEN MIT EXPOSITION GEGENÜBER BIOLOGISCHEM MATERIAL, DIE VON 2015 BIS 2019 BEI ARBEITNEHMERN IM BUNDESSTAAT AMAPÁ, AMAZONAS, BRASILIEN, AUFGETRETEN SIND.
}

\section{ORIGINALER ARTIKEL}

MACIEL, Danilo Pereira Garcia ${ }^{1}$, MEDEIROS, José Leandro Tomaz², SILVA, Mariana Freitas $\mathrm{da}^{3}$, SILVA, Matheus Freitas da ${ }^{4}$, FECURY, Amanda Alves ${ }^{5}$, DIAS, Claudio Alberto Gellis de Mattos $^{6}$, OLIVEIRA, Euzébio de ${ }^{7}$, DENDASCK, Carla Viana $^{8}$, DAHER, Donizete Vago ${ }^{9}$, ARAÚJO, Maria Helena Mendonça de ${ }^{10}$

MACIEL, Danilo Pereira Garcia. Et al. Epidemiologisches Profil von Unfällen mit Exposition gegenüber biologischem Material, die von 2015 bis 2019 bei Arbeitnehmern im Bundesstaat Amapá, Amazonas, Brasilien, aufgetreten sind. Revista Científica Multidisciplinar Núcleo do Conhecimento. Jahr 06, Ed. 03, Vol. 04, pp. 127-141. März 2021. ISSN: 2448-0959, Zugangslink: https://www.nucleodoconhecimento.com.br/gesundheit/biologischem-material, DOI: 10.32749/nucleodoconhecimento.com.br/gesundheit/biologischem-material

\footnotetext{
${ }^{1}$ Student im Medizinstudium an der Bundesuniversität Amapá (UNIFAP).

${ }^{2}$ Student im Medizinstudium an der Bundesuniversität Amapá (UNIFAP).

${ }^{3}$ Student im Medizinstudium an der Bundesuniversität Amapá (UNIFAP).

${ }^{4}$ Student im Medizinstudium an der Staatlichen Universität von Pará (UEPA).

${ }^{5}$ Biomedizin, $\mathrm{PhD}$ in topischen Krankheiten, Professor und Forscher des medizinischen Kurses am Campus Macapá der Bundesuniversität Amapá (UNIFAP).

${ }^{6}$ Biologe, Doktor in Verhaltenstheorie und -forschung, Professor und Forscher des Chemie-Studiengangs am Institut für grundlegende, technische und technologische Ausbildung von Amapá (IFAP) und des Graduiertenkollegs für berufliche und technologische Ausbildung (PROFEPT IFAP).

${ }^{7}$ Biologe, PhD in Topical Diseases, Professor und Forscher des Sportlehrgangs an der Federal University of Pará (UFPA).

${ }^{8}$ Theologe, PhD in klinischer Psychoanalyse. Er hat 15 Jahre mit Scientific Methodology (Research Method) in der Orientierung der wissenschaftlichen Produktion von Master- und Doktoranden gearbeitet. Spezialist für Marktforschung und Forschung mit Schwerpunkt Gesundheit.

${ }^{9}$ Krankenschwester und Geburtshelfer, PhD in Public Health (FCM-UNICAMP), Professor und Forscher an der Universidade Federal Fluminense (UFF).

${ }^{10}$ Arzt, Master in Lehre und Gesundheitswissenschaften. Professor und Forscher an der Bundesuniversität Amapá (UNIFAP), Campus Macapá, AP.
}

RC: 78404

Disponível em: https://www.nucleodoconhecimento.com.br/gesundheit/biologischemmaterial 


\section{ABSTRAKT}

Unbeabsichtigte Exposition gegenüber scharfen Instrumenten sind die häufigsten Arbeitsunfälle, an denen Fachkräfte und Studenten in einem Krankenhausumfeld beteiligt sind. Biologisches Material (MB) entsteht durch direkten Kontakt zwischen Blut und genitalen oder serösen organischen Flüssigkeiten mit ungesunder Haut, Schleimhäuten oder durch direkte perkutane Inokulation durch scharfe Gegenstände.Ziel dieser Arbeit war es, das epidemiologische Profil von Unfällen mit Exposition gegenüber biologischem Material $\mathrm{zu}$ charakterisieren, die bei Arbeitnehmern im brasilianischen Bundesstaat Amapá, Amazonas, im Zeitraum von 2015 bis 2019 aufgetreten sind, und die Anzahl, Art der Vorkommen, den Beruf und die Umstände zu analysieren des Unfalls. Eine retrospektive, deskriptive epidemiologische Querschnittsstudie wurde mit einem quantitativen Ansatz durchgeführt. So wurde die Datenbank des Informationssystems für meldepflichtige Krankheiten (SINAN) nach Aufzeichnungen durchsucht, die sich auf Meldungen von Unfällen mit biologischem Material beziehen, die im Bundesstaat Amapá im Zeitraum von 2015 bis 2019 aufgetreten sind und vom Arbeitsmedizinischen Überwachungszentrum registriert wurden ( NVST) / Referenzzentrum für Arbeitsmedizin (CEREST / AP). Die berufliche Exposition gegenüber biologischem Material birgt ein potenzielles Risiko für die Übertragung von Krankheiten. Die Anweisung der Arbeitnehmer, Unfälle unverzüglich zu melden, ist für die Bereitstellung des Benachrichtigungssystems von wesentlicher Bedeutung. In Bezug auf die Umstände waren die beiden am häufigsten festgestellten Ursachen nahe beieinander liegende Werte, die Verabreichung von Medikamenten (21,6\%) und die unzureichende Entsorgung des Materials (20\%). Diese falsche Entsorgung zeigt den mangelnden Eifer der Fachkräfte für ihre eigene Gesundheit.

Schlüsselwörter: Biologische Unfälle, Exposition, Epidemiologie, Amazonas, Arbeiter.

$\mathrm{RC}: 78404$

Disponível em: https://www.nucleodoconhecimento.com.br/gesundheit/biologischemmaterial 


\section{EINFÜHRUNG}

Unbeabsichtigte Expositionen mit scharfen Instrumenten sind die häufigsten Arbeitsunfälle, an denen Fachkräfte und Studenten in einem Krankenhausumfeld beteiligt sind. Schätzungen zufolge ereignen sich nach Angaben der CDC (Centers for Diseases Control and Prevention) (RUAS et al., 2012; CUNHA te al., 2019) jährlich etwa 385.000 Fälle von scharfen Unfällen, an denen Angehörige der Gesundheitsberufe in Krankenhäusern auf der ganzen Welt beteiligt sind.

Ein Unfall mit biologischem Material (MB) resultiert aus dem direkten Kontakt zwischen Blut und genitalen oder serösen organischen Flüssigkeiten, mit ungesunder Haut, Schleimhäuten oder durch direkte perkutane Inokulation durch scharfe Gegenstände. Die berufliche Exposition tritt während der Ausübung der Arbeit auf, entweder bei Dienstleistungen zur Gesundheitsversorgung der Bevölkerung oder während der Abfallsammlung oder einer anderen ausgeführten Arbeitsfunktion und ist Bestandteil der Nationalen Liste der obligatorischen Meldekrankheiten (BRASIL, 1991; DAROUICHE et al. , 2014; ARAÚJO et al., 2019).

Unter den mehr als zwanzig Krankheitserregern, die an versehentlichen Expositionen beteiligt sind, heben wir das Hepatitis B-Virus (HBV), das Hepatitis C-Virus (HCV) und das Human Immunodeficiency Virus (HIV) hervor, da das Übertragungsrisiko auf $6 \%$ und 30 geschätzt wird \% für HBV; zwischen $5 \%$ und $10 \%$ für HCV und $0,3 \%$ für HIV nach einer Verletzung mit einem Piercing-Objekt (CDC, 2001). Zusätzlich zu viralen Pathogenen verursacht das ätiologische Mittel Clostridium tetani einen versehentlichen Tetanus durch Inokulation von Sporen auf Schleimhäuten und ungesunder Haut, insbesondere bei tiefen oberflächlichen Wunden, bei denen Materialien mit falscher Entsorgung verwendet werden (BRASIL, 2018; SILVA et al., 2020) . Das Risiko einer Infektion durch diese Exposition hängt von folgenden Faktoren ab: Ausmaß der Läsion, Volumen der vorhandenen biologischen Flüssigkeit, systemische Bedingungen des Opfers, Merkmale der vorhandenen

RC: 78404

Disponível em: https://www.nucleodoconhecimento.com.br/gesundheit/biologischem$\underline{\text { material }}$ 
Mikroorganismen, serologischer Status der Quellperson sowie Verhalten durchgeführt nach der Ausstellung (BRASIL, 2017, VASCONCELOS et al., 2020).

Die Verordnung Nr. 1061/2020 legt fest, dass Arbeitsunfälle mit Exposition gegenüber biologischem Material obligatorisch sind und wöchentlich in das Informationssystem für meldepflichtige Krankheiten (SINAN) aufgenommen werden müssen. Zum Zeitpunkt der Unfallteilnahme muss das individuelle Meldeformular (FNI) ausgefüllt werden. Es sollte jedoch nicht darauf gewartet werden, dass das FNI ausgefüllt wird, um den Arbeitnehmer zu unterstützen (BRASIL, 2020).

Der Hauptweg, um eine Exposition zu vermeiden, ist die vorbeugende Aufklärung und die ordnungsgemäße Verwendung von persönlicher Schutzausrüstung (PSA) unter Einhaltung der Standards für die biologische Sicherheit. Es ist wichtig, den serologischen Status sowohl der exponierten Person als auch der Quellperson zu bewerten. Die Postexpositionsprophylaxe (PEP) sollte in den ersten Stunden des Unfalls eingeleitet werden, wobei die antiretrovirale HIV-Therapie (ART) als PEP für HIV angegeben wird und eine ergänzende Impfung oder passive Immunisierung gegen Virushepatitis und Tetanus empfohlen wird (BRASIL, 2017).

\section{ZIELSETZUNG}

Charakterisierung des epidemiologischen Profils von Unfällen mit Exposition gegenüber biologischem Material, die bei Arbeitnehmern im Bundesstaat Amapá, Amazonas, Brasilien, im Zeitraum von 2015 bis 2019 aufgetreten sind, Analyse von Anzahl, Art des Auftretens, Beruf und Umständen des Unfalls.

\section{METHODE}

Eine retrospektive, deskriptive epidemiologische Querschnittsstudie wurde mit einem quantitativen Ansatz durchgeführt. So wurde die Datenbank des Informationssystems für meldepflichtige Krankheiten (SINAN) nach Aufzeichnungen durchsucht, die sich

$\mathrm{RC}: 78404$

Disponível em: https://www.nucleodoconhecimento.com.br/gesundheit/biologischemmaterial 
auf Meldungen von Unfällen mit biologischem Material beziehen, die im Bundesstaat Amapá im Zeitraum von 2015 bis 2019 aufgetreten sind und vom Arbeitsmedizinischen Überwachungszentrum registriert wurden ( NVST) / Referenzzentrum für Arbeitsmedizin (CEREST / AP).

In diesem Sinne umfassten die analysierten Variablen die Gesamtzahl der Unfälle, die Anzahl der pro Jahr getrennten Fälle, den Unfallort, das biologische Geschlecht und den Beruf des Verletzten.

Die Zahlen wurden im Oktober 2020 durch den Export von Aufzeichnungen aus SINAN durch das von DATASUS / Gesundheitsministerium entwickelte TabwinProgramm in Tabellenkalkulationen des Microsoft Excel-Programms ermittelt. Anschließend wurden sie zusammengestellt, tabelliert und analysiert, in absoluten und relativen Häufigkeiten berechnet und ausgedrückt und schließlich zusammen mit der untersuchten Literaturforschung analysiert. Aufgrund des öffentlichadministrativen Bereichs der Sekundärdaten war die Stellungnahme der Forschungsethikkommission gemäß Resolution 466-2012 nicht erforderlich.

\section{ERGEBNISSE}

Im Fünfjahreszeitraum 2015-2019 wurden in SINAN 860 Fälle von Unfällen mit biologischem Material gemeldet. Das Jahr, in dem die meisten Unfälle auftraten, war 2016 (199 Fälle = 23,1\%), im Gegensatz zum Vorjahr, in dem 2015 die niedrigste Rekordrate verzeichnet wurde (128 Fälle $=14,8 \%$ ) (Abbildung 1).

RC: 78404

Disponível em: https://www.nucleodoconhecimento.com.br/gesundheit/biologischemmaterial 
Abbildung 1 zeigt die Anzahl der Arbeitsunfälle mit Exposition gegenüber biologischem Material im Bundesstaat Amapá, Amazonas, Brasilien zwischen 2015 und 2019.

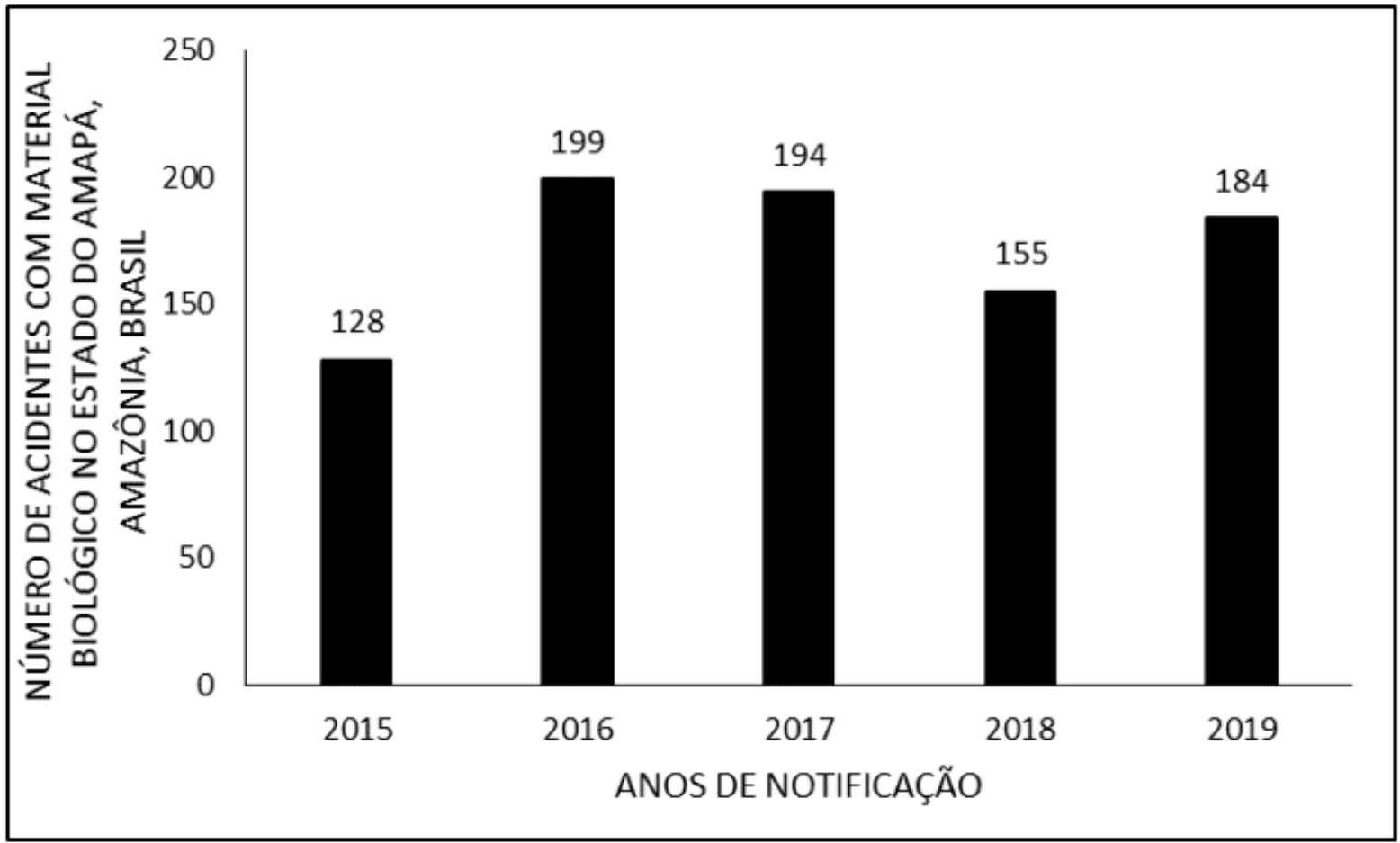

Quelle: SINAN, 2020.

Das weibliche Geschlecht mit 595 Fällen (69,1\%) überwog das männliche, das weniger als ein Drittel der registrierten Fälle ausmachte, 265 (30,8\%) (Abbildung 2).

RC: 78404

Disponível em: https://www.nucleodoconhecimento.com.br/gesundheit/biologischem$\underline{\text { material }}$ 
Abbildung 2 zeigt die Anzahl der Arbeitsunfälle mit Exposition gegenüber biologischem Material im Bundesstaat Amapá, Amazonas, Brasilien, zwischen 2015 und 2019 nach Geschlecht (Geschlecht).

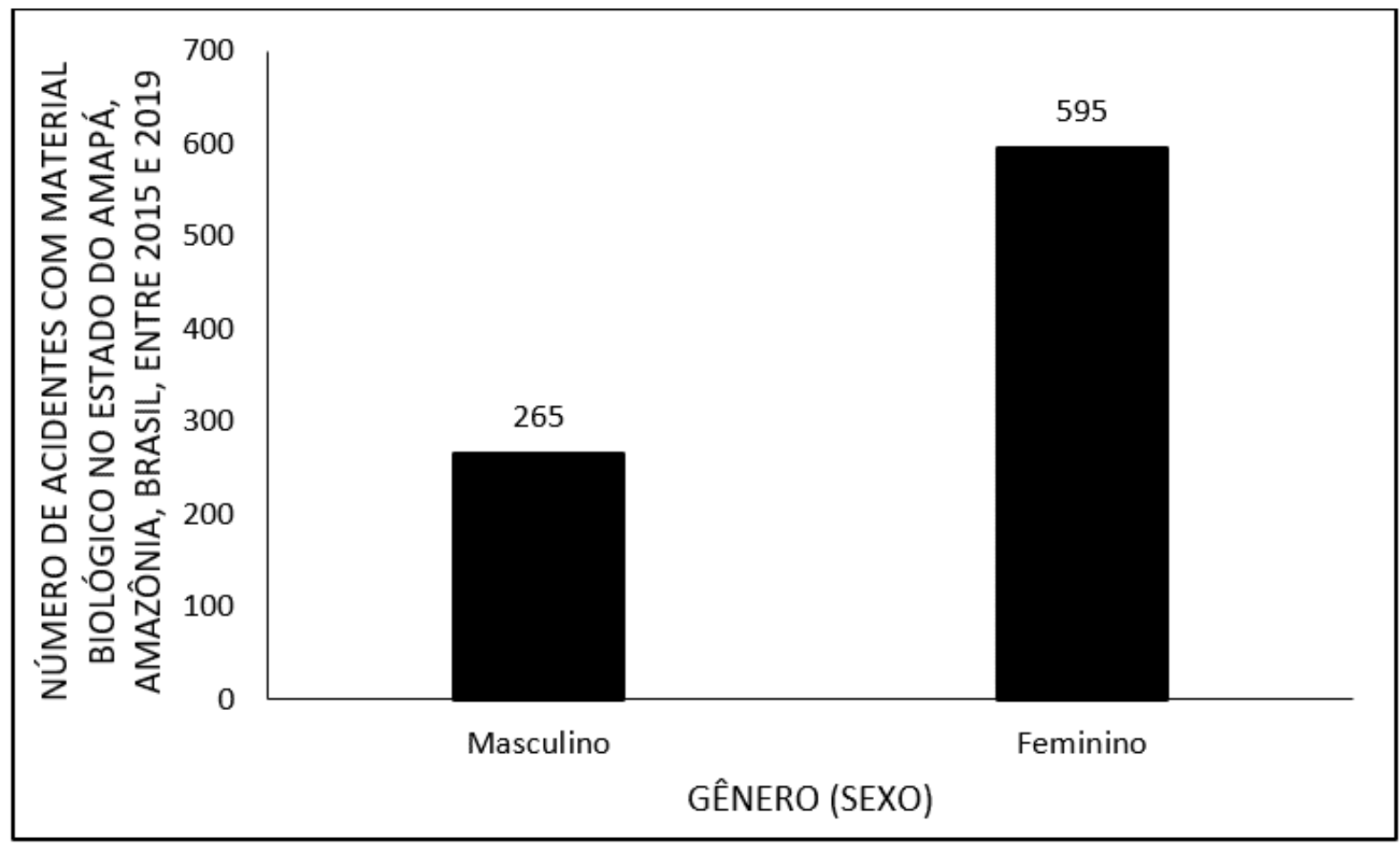

Quelle: SINAN, 2020.

In Bezug auf die berufliche Tätigkeit war die Klasse der am stärksten von Unfällen betroffenen Personen die der Pflegefachkräfte (346 Fälle =40,13\%), verteilt auf: 300 Pflegetechniker (34,9\%), 45 Krankenschwestern (5,23\%). Der zweite Platz wird von Studenten mit 116 Fällen (13,4\%) belegt. Gefolgt von den Sanitärservern (Hausmeister, Müllsammler und Straßenreiniger) mit insgesamt 66 Fällen (7,8\%). Die Ärzteschaft hat 35 Fälle (4,06\%), in denen alle Fachgebiete hinzugefügt werden, wobei die höchste Inzidenz bei klinischen Ärzten (1,86\%) und Allgemeinchirurgen $(1,04 \%) \mathrm{zu}$ verzeichnen ist. Zahnärzte stellen 24 Fälle dar (2,7\%), Zahnhygienetechniker haben jedoch im Vergleich zu Zahnärzten eine höhere Anzahl von Unfällen als 53 Fälle (6,16\%). Apotheker, Physiotherapeuten und Biomedizin machen zusammen 1,62\% aller Fälle aus (Tabelle 1).

$\mathrm{RC}: 78404$

Disponível em: https://www.nucleodoconhecimento.com.br/gesundheit/biologischem$\underline{\text { material }}$ 


\begin{tabular}{c|c|c|c|c|c|c}
\cline { 2 - 7 } & $\mathbf{2 0 1 5}$ & $\mathbf{2 0 1 6}$ & $\mathbf{2 0 1 7}$ & $\mathbf{2 0 1 8}$ & $\mathbf{2 0 1 9}$ & TOTAL \\
\hline $\begin{array}{c}\text { Profissionais de } \\
\text { enfermagem }\end{array}$ & 73 & 70 & 76 & 65 & 62 & 346 \\
\hline $\begin{array}{c}\text { Estudantes } \\
\text { Gari, faxineiro, coletor de } \\
\text { lixo }\end{array}$ & 10 & 27 & 32 & 22 & 25 & 116 \\
\hline $\begin{array}{c}\text { Profissionais médicos } \\
\text { Cirurgião dentista }\end{array}$ & 3 & 13 & 8 & 4 & 7 & 35 \\
\hline $\begin{array}{c}\text { Outros profissionais de } \\
\text { saúde }\end{array}$ & 4 & 10 & 6 & 9 & 24 & 53 \\
\hline $\begin{array}{c}\text { Profissionais de outras } \\
\text { áreas }\end{array}$ & 14 & 71 & 52 & 29 & 32 & 198 \\
\hline TOTAL & $\mathbf{9 1}$ & $\mathbf{1 2 1}$ & $\mathbf{1 2 3}$ & $\mathbf{1 1 1}$ & $\mathbf{1 2 8}$ & $\mathbf{8 3 8}$ \\
\hline
\end{tabular}

Quelle: SINAN, 2020.

Tabelle 1 zeigt die Anzahl der Arbeitsunfälle mit Exposition gegenüber biologischem Material im Bundesstaat Amapá, Amazonas, Brasilien, zwischen 2015 und 2019 in Bezug auf die Beschäftigung.

In Anbetracht der unterschiedlichen Umstände des Unfalls war die häufigste Ursache für Verletzungen mit biologischem Material die Verabreichung von Medikamenten $(21,6 \%)$, gefolgt von einer unzureichenden Entsorgung des Materials $(20 \%)$ sowie chirurgischen und zahnärztlichen Eingriffen (15\%). Zwei weitere bemerkenswerte Ursachen waren die Handhabung der Entsorgungsbox für scharfe Gegenstände $(3,9 \%)$ und das Wiederverschließen der Nadeln (2,32\%) (Tabelle 2).

RC: 78404

Disponível em: https://www.nucleodoconhecimento.com.br/gesundheit/biologischem$\underline{\text { material }}$ 
Tabelle 2 zeigt die Anzahl der Arbeitsunfälle mit Exposition gegenüber biologischem Material im Bundesstaat Amapá, Amazonas, Brasilien, zwischen 2015 und 2019, je nach Anlass.

\begin{tabular}{|c|c|c|c|c|c|c|}
\hline & 2015 & 2016 & 2017 & 2018 & 2019 & Total \\
\hline $\begin{array}{c}\text { Administração } \\
\text { endovenosa }\end{array}$ & 22 & 23 & 21 & 15 & 22 & 103 \\
\hline $\begin{array}{l}\text { Descarte inadequado } \\
\text { lixo }\end{array}$ & 20 & 17 & 21 & 18 & 18 & 94 \\
\hline $\begin{array}{c}\text { Descarte inadequado } \\
\text { chão }\end{array}$ & 13 & 16 & 26 & 9 & 14 & 78 \\
\hline $\begin{array}{l}\text { Procedimento } \\
\text { odontológico }\end{array}$ & 2 & 20 & 13 & 17 & 23 & 75 \\
\hline $\begin{array}{l}\text { Administração } \\
\text { intramuscular }\end{array}$ & 5 & 8 & 12 & 21 & 15 & 61 \\
\hline Procedimento cirúrgico & 4 & 21 & 12 & 4 & 13 & 54 \\
\hline Punção coleta & 9 & 7 & 5 & 6 & 9 & 36 \\
\hline $\begin{array}{c}\text { Manipulação de caixa } \\
\text { perfuro/cortante }\end{array}$ & 4 & 6 & 9 & 9 & 6 & 34 \\
\hline Lavagem de material & 6 & 4 & 8 & 3 & 10 & 31 \\
\hline $\begin{array}{c}\text { Procedimento } \\
\text { laboratorial }\end{array}$ & 2 & 2 & 4 & 5 & 10 & 23 \\
\hline Punção NE & 6 & 5 & 4 & 3 & 2 & 20 \\
\hline Lavanderia & 2 & 15 & 0 & 2 & 1 & 20 \\
\hline Reencape & 2 & 6 & 2 & 5 & 5 & 20 \\
\hline $\begin{array}{c}\text { Administração } \\
\text { subcutânea }\end{array}$ & 0 & 7 & 6 & 0 & 1 & 14 \\
\hline Dextro & 7 & 0 & 4 & 0 & 1 & 12 \\
\hline $\begin{array}{l}\text { Administração } \\
\text { intradérmica }\end{array}$ & 3 & 3 & 2 & 0 & 0 & 8 \\
\hline Ignorado/Branco & 4 & 10 & 11 & 11 & 2 & 38 \\
\hline Outros & 17 & 29 & 34 & 27 & 32 & 139 \\
\hline Total & 128 & 199 & 194 & 155 & 184 & 860 \\
\hline
\end{tabular}

Quelle: SINAN, 2020.

RC: 78404

Disponível em: https://www.nucleodoconhecimento.com.br/gesundheit/biologischem$\underline{\text { material }}$ 


\section{DISKUSSION}

Anhand der vorgelegten Daten kann überprüft werden, ob Frauen die Hauptbeschäftigten bei Unfällen mit biologischem Material sind (69,1\%). Eine Analyse einer historischen Reihe, die im Format eines epidemiologischen Bulletins veröffentlicht wurde, das zwischen 2009 und 2020 in Rio de Janeiro durchgeführt wurde, ergab einen ungefähren Anteil von einem Mann pro drei Frauen, die einen Unfall erleiden (Brasil, 2020; CARNEIRO et al ., 2020). Ein ähnliches Ergebnis wurde in der Studie von Khalil et al. (2015) gefunden, die in einem spezialisierten Pflegedienst im Südosten Brasiliens durchgeführt wurde, in der Frauen dreimal häufiger Unfälle erlitten als Männer.

Unter den analysierten Berufen wurde die Kategorie der Angehörigen der Gesundheitsberufe $(60,11 \%)$ als Hauptopfer angegeben. Dies ist darauf zurückzuführen, dass diese Fachkräfte in direktem Kontakt mit Patienten stehen, Verfahren durchführen und mit potenziell kontaminierten Materialien umgehen (MOREIRA et al. , 2020). Diese Ergebnisse entsprachen Studien, die in anderen Ländern wie Ägypten und Italien durchgeführt wurden (TALAAT et al., 2003; MAIDA et al., 2020).

Nach den Ergebnissen der Bildschirmuntersuchungen waren Pflegefachkräfte die am stärksten an Unfällen beteiligte Kategorie, gefolgt von Studenten, was die Feststellung von Santos (2015) bestätigt, der in seinen Untersuchungen zeigte, dass Pflegetechniker, gefolgt von Medizinstudenten, am stärksten von scharfen Gegenständen betroffen waren Unfälle. In diesem Sinne gibt es auch die Studie von Kon et al (2011) in einem Krankenhaus in Curitiba (PR), in der die meisten Unfälle Pflegehelfer (30,1\%), Pflegetechniker $(15 \%, 2)$ und Studenten $(10,8 \%)$ betrafen. benachrichtigt und bei SINAN registriert.

Figueiredo (2018), der die an Unfällen mit biologischem Material beteiligten Fachkräfte analysierte, stellte fest, dass 47\% der analysierten Fälle im Pflegebereich

$\mathrm{RC}: 78404$

Disponível em: https://www.nucleodoconhecimento.com.br/gesundheit/biologischemmaterial 
arbeiteten; 19\% waren Studenten, 6\% waren Ärzte; 6\% Zahnärzte, 4\% waren Mitarbeiter in den Bereichen Sammlung, Abfall, Reinigung und Konservierung von öffentlichen Bereichen. Zur Bestätigung dieser Daten erscheinen zuerst Pflegetechniker mit mehr als einem Drittel der veröffentlichten Unfallfälle. Diese Fachkräfte stellen die größte Anzahl von Arbeitnehmern in den Diensten dar, die Patienten direkt unterstützen (NEIVA et al., 2019). Oliveira et al. (2015), veröffentlichten eine epidemiologische Studie über MB-Unfälle mit $\mathrm{MB}$ bei Pflegefachkräften in einer Stadt in Ceará. Von 277 Unfallberichten waren 217 Pflegehelfer (78,3\%), was bestätigt, dass diese Fachkräfte am stärksten Unfällen ausgesetzt sind.

Bei Unfällen mit biologischem Material konfigurieren die Studierenden die zweitwichtigste Kategorie, was $13,4 \%$ der insgesamt analysierten Personen entspricht. Dies kann auf die Tatsache zurückzuführen sein, dass sie eine Schulung und Ausbildung absolvieren und daher professionelle Unerfahrenheit bei der technischen Durchführung von Verfahren und beim Umgang mit Material zeigen. Reis et al. (2013) führten eine epidemiologische Studie über Unfälle mit biologischem Material in einer chirurgischen Notaufnahme unter Beteiligung von Medizinstudenten durch und stellten fest, dass unter den 100 teilnehmenden Studenten 2012 ein Prozentsatz von 32\% diese Art von Unfall erlitt. Die wichtigsten in dieser Studie identifizierten Faktoren Es mangelte an Schulung und es wurde keine persönliche Schutzausrüstung verwendet.

Es ist auch wichtig, die Fachleute hervorzuheben, die in der Reinigung arbeiten, da sie die dritte Gruppe mit dem höchsten Risiko einer Kontamination mit biologischem Material darstellen, da sie direkten Kontakt mit organischen Abfällen und Krankenhausabfällen haben (MATOS et al., 2019). Sie werden von Müllsammlern, Hausmeistern und Straßenreinigern vertreten. Canini; Gir und Machado (2005) entwickelten eine Studie, in der Mitarbeiter des Kundendienstes, die Unfälle mit einem potenziellen Kontaminationsrisiko erlitten hatten, etwa vier Jahre lang in einem

$\mathrm{RC}: 78404$

Disponível em: https://www.nucleodoconhecimento.com.br/gesundheit/biologischemmaterial 
tertiären Universitätskrankenhaus in São Paulo nachverfolgt wurden. Es wurde festgestellt, dass die Mehrheit (94,9\%) der Unfälle die Quellperson nicht entdecken konnte, was PEP bei Verwendung von ART erforderte, und dies lag an der falschen Entsorgung von Nadeln.

Aufgrund der Ergebnisse dieser Daten gibt es einen Unterschied in der Anzahl der Unfälle im Vergleich zu verschiedenen Kategorien von Angehörigen der Gesundheitsberufe. Ärzte (4,06\%) haben im Bundesstaat Amapá eine höhere Inzidenz als Zahnärzte (2,7\%). Dementsprechend wurde in der Studie von Reis, Gómez, Diniz (2019) festgestellt, dass Ärzte eine höhere Inzidenz hatten als Zahnärzte, was durch die bessere Einhaltung der üblichen Vorsichtsmaßnahmen erklärt wurde. Das gleiche Muster wurde bei Medizinstudenten im Vergleich zur Zahnmedizin wiederholt - bessere Einhaltung der Standardvorkehrungen.

Laut Brozoski (2010) ereignet sich der Unfall mit biologischem Material während der Praxis in chirurgischen Disziplinen im zahnmedizinischen Kurs mit Anästhesienadelmaterial, das subkutan und gingiv ist. Die Daten zeigen, dass der Zahnarzt mit 24 Fällen im Berichtszeitraum auf dem fünften Platz liegt und bei der Verabreichung von Medikamenten auf intradermalem Weg nur 8 Fälle gemeldet wurden, was die Möglichkeit schafft, eine Hypothese der Unterberichterstattung in Betracht zu ziehen, die in mehr als 95\% der Fälle auftreten kann Fälle nach Santos (2015).

Wenn man sich mit dem Unterschied bei Unfällen mit MB zwischen Ärzten klinischer und chirurgischer Fachgebiete konfrontiert sieht, überwiegt in Amapá der erste gegenüber dem zweiten. Im Gegensatz zu dem, was in einem Universitätskrankenhaus in Kolumbien beobachtet wurde, wo Ärzte in chirurgischen Bereichen 3,3-mal mehr Unfälle hatten als Ärzte.

Bewohner klinischer Gebiete. (TAPIAS, TAPIAS, TORRES, 2007). Dieser Unterschied ist möglicherweise auf die mangelnde Einheitlichkeit der Prävention

$\mathrm{RC}: 78404$

Disponível em: https://www.nucleodoconhecimento.com.br/gesundheit/biologischem$\underline{\text { material }}$ 
zwischen Ort und Arbeit zurückzuführen, obwohl es eindeutige Regeln gibt (MATOS et al., 2019).

In Bezug auf die Umstände waren die beiden am häufigsten festgestellten Ursachen nahe beieinander liegende Werte, die Verabreichung von Medikamenten $(21,6 \%)$ und die unzureichende Entsorgung des Materials (20\%). Diese falsche Entsorgung zeigt den mangelnden Eifer der Fachkräfte für ihre eigene Gesundheit. Cordeiro et al. (2016) fanden sehr ähnliche Ergebnisse, die auch die Verabreichung von Medikamenten $(9,7 \%)$ und die unangemessene Materialentsorgung $(9,5 \%)$ in ihren 2012 im Bundesstaat Bahia durchgeführten Untersuchungen mit Daten aus 1613 SINAN-Fällen betrafen. Im Gegensatz dazu beobachteten Janjua, Khan, Mahmood (2010) in ihrer Studie, dass das Wiederverschließen von Nadeln die häufigste Unfallursache war, da sie das Risiko einer perkutanen Exposition um das Zweifache erhöhte.

\section{SCHLUSSFOLGERUNGEN}

Die berufliche Exposition gegenüber biologischem Material birgt ein potenzielles Risiko für die Übertragung von Krankheiten. In Bezug auf Meldungen und vorbeugende Maßnahmen bei Arbeitsunfällen, an denen Arbeitnehmer in Amapá beteiligt sind, sind noch einige Herausforderungen zu bewältigen.

Die Anweisung der Arbeitnehmer, Unfälle unverzüglich zu melden, ist für die Bereitstellung des Benachrichtigungssystems von wesentlicher Bedeutung. Die im Benachrichtigungsformular korrekt ausgefüllten Daten sind wichtig, um das Auftreten von Unfällen zu überwachen, eine frühzeitige Nachverfolgung zu gewährleisten, um das Risiko von Infektionen zu verringern, Aktivitäten mit höherem Risiko zu identifizieren, die Überwachung zu verstärken und die Biosicherheitspraktiken zu verbessern und die beruflichen Risiken zu verringern Risiken.

RC: 78404

Disponível em: https://www.nucleodoconhecimento.com.br/gesundheit/biologischem$\underline{\text { material }}$ 
Die wichtigsten Möglichkeiten zur Verhinderung von Arbeitsunfällen sind die Einhaltung und Befolgung von Sicherheitsmaßnahmen wie die ordnungsgemäße Verwendung von PSA, Vorsicht bei der Verabreichung von Medikamenten, korrekte Entsorgung von Materialien, vollständiger und aktualisierter Impfplan für Hepatitis B und Tetanus sowie Exposition nach Prophylaxe mit angemessener Überwachung . Die Prävention dieser Infektionen ist eine wichtige Säule der Arbeitssicherheit.

\section{VERWEISE}

ARAÚJO, A. F. B. et al. Internações por acidentes de trânsito no Estado do Amapá entre os anos 2014-2018 Revista Científica Multidisciplinar Núcleo do Conhecimento, v. 1 , p. 1-10, 2019. Disponível em:< https://www.nucleodoconhecimento.com.br/saude/internacoes-por-acidentes >.

BRASIL. Portaria no 1061, de 18 de maio de 2020. Revoga A Portaria № 264, de 17 de Fevereiro de 2020, e Altera A Portaria de Consolidação № 4/gm/ms, de 28 de Setembro de 2017, Para Incluir A Doença de Chagas Crônica, na Lista Nacional de Notificação Compulsória de Doenças, Agravos e Eventos de Saúde Pública nos Serviços de Saúde Públicos e Privados em Todo $O$ Território Nacional. 102. ed. Brasília, DF: Ministério da Saúde, 29 maio de 2020. Seção 1, p. 229.

BRASIL. Constituição (1990). Lei no 8213/91, de 24 de julho de 1991. Brasília, DF. 1991. Disponível em: http://www.planalto.gov.br/ccivil_03/leis//8213cons.htm. Acesso em: 18 fev. 2020.

BRASIL. Ministério da Saúde. Protocolo Clínico e Diretrizes Terapêuticas para Hepatite B e Coinfecções. Brasília: Secretaria de Vigilância em Saúde, Departamento de DST, Aids e Hepatites Virais, Ministério da Saúde, 2017.

RC: 78404

Disponível em: https://www.nucleodoconhecimento.com.br/gesundheit/biologischem$\underline{\text { material }}$ 
BRASIL. Ministério da Saúde. Guia de Vigilância em Saúde: volume único. 2a edição. Brasília: Secretaria de Vigilância em Saúde, Coordenação-Geral de Desenvolvimento da Epidemiologia em Serviços. Ministério da Saúde, 2017.

BRASIL. Ministério da Saúde. Protocolo clínico e diretrizes terapêuticas para profilaxia pós-exposição (PEP) de risco à infecção pelo HIV, IST e hepatites virais. Brasília: Ministério da Saúde, 2017, p 164-172, volume único. Disponível em: Volume-Unico-2017.pdf (saude.gov.br) . Acesso em :10/02/2020.

BRASIL. Ministério da Saúde. Boletim Epidemiológico: Situação epidemiológica do tétano acidental no Brasil, 2007-2016. Volume 49/ Jun. 2018. Brasília: Secretaria de Vigilância em Saúde, 2018.

BROZOSKI, M.A.; Traina A.A.; NACLÉRIO-HOMEM, M.G.; DEBONI, M.C.Z. Ocorrência de acidentes perfurocortantes em um curso de odontologia. Rev Gaúcha Odontol, Porto Alegre: vol.58 n.1, p 77-80, 2010.

CANINI S.R.M.S.; GIR, E.; MACHADO, A. A. Accidents with potentially hazardousbiological material among workers in hospital supporting services. Revista Latino-Americana de Enfermagem, Ribeirão Preto, v. 13, n. 4, p. 496-500, jul/ago, 2005.

CARNEIRO, L. Q. C. et al. Perfil epidemiológico dos pacientes atendidos devido a acidentes ofídicos no norte do Brasil, Região Amazônica, no período de 2009 a 2019. Revista Científica Multidisciplinar Núcleo do Conhecimento, v. 3, p. 1-19, 2020. Disponível em: < https://www.nucleodoconhecimento.com.br/saude/acidentesofidicos $>$.

CDC. Guidelines for the management of occupational exposures to HBV, HCV, and HIV and recommendations for postexposure prophylaxis. Atlanta: MMWR Recomm Rep, v50(RR11),P 1-52, 2001.

RC: 78404

Disponível em: https://www.nucleodoconhecimento.com.br/gesundheit/biologischem$\underline{\text { material }}$ 
CORDEIRO, T. M. S. C.; NETO, J. N. C.; CARDOSO, M. C. B. C.; MATTOS, A. I. S.; SANTOS, K. O. B.; ARAÚJO, T. M. Acidentes de trabalho com exposição à material biológico: Descrição dos casos na Bahia. Rev. Epidemiol. Control. Infec, Santa Cruz do Sul, v. 6, n. 2, p. 50-56, 2016.

CUNHA, A. A. et al. Tendência na incidência de acidentes de trajeto em trabalhadores no Brasil entre 2009 e 2016. Bras Med Trab., v. 17, n. 4, p. 490-498, 2019. Disponível em: < http://rbmt.org.br/about-the-authors/489/pt-BR >.

DAROUICHE, M.H., et al. Occupational blood exposure among health care personnel and hospital trainees. Int J Occup Environ Med. [S.I], v.5, n 1, p 57-61, 2014.

FIGUEIREDO, W. M.; et al. Acidentes ocupacionais por material de risco biológico: estudo etnográfico. Braz. J. of Develop, Curitiba, v. 4, n. 7, Edição Especial, p. 4500-4518, nov. 2018.

JANJUA, N.Z.; KHAN, M.I.; MAHMOOD, B. Sharp injuries and their determinants among health care workers at first-level care facilities in Sindh Province, Pakistan. Trop Med Int Health, [S.I] v15, p 1244-1251, 2010.

KHALIL, S.S.; KHALIL, O.A.K.; LOPES-JÚNIOR, L.C.; CABRAL, D.B.; BOMFIM, E.O.; LANDUCCI, L.F.; SANTOS, M.L.S.G. Occupational exposure to bloodborne pathogens in a specialized care service in Brazil. American Journal of Infection Control, [S.I], v43, n8, p. e39-e41, 2015.

KON, N.M.; SOLTOSKI, F.; REQUE JÚNIOR. M.; LOZOVEY, J.C.A. Acidentes de trabalho com material biológico em uma Unidade Sentinela: casuística de 2.683 casos. Rev Bras Med Trab. [S.I], v 9, n 1, p 33-38, 2011.

MAIDA, C.M.; APREA, L.; CALAMUSA, G.; CAMPISI, F.; FAVARO, D.; FIORINO, G.R.; FODALE, A.M.; MANIGLIA, M.L.; MARCHESE, V.; VELARDO, M.M.;

RC: 78404

Disponível em: https://www.nucleodoconhecimento.com.br/gesundheit/biologischem$\underline{\text { material }}$ 
Torregrossa M.V. Blood and body fluids exposure of healthcare workers in a university hospital of Palermo, Italy: a fourteen years long surveillance. Annali di Igiene, [S.I], Volume 32, n6, 2020.

MATOS, D. V. D. et al. Caracterização epidemiológica dos indivíduos que sofreram acidentes de trabalho nas macrorregiões brasileiras, nos anos de 2016 a 2018. Revista Científica Multidisciplinar Núcleo do Conhecimento, v. 1, 2019. Disponível em: < https://www.nucleodoconhecimento.com.br/saude/acidentes-detrabalho >.

MOREIRA, E. C. D. M. et al. Impactos diretos e indiretos na neurocognição humana, decorrentes da intoxicação por metilmercúrio e seus agravos para a saúde coletiva. Research, Society and Development, v. 912, p. e4409128005, 2020. Disponível em: < https://rsdjournal.org/index.php/rsd/article/view/8005 >.

NEIVA, C. A. C. et al. Caracterização epidemiológica das intoxicações exógenas por substâncias nocivas e acidentes por animais peçonhentos em crianças no Estado do Amapá. Revista Científica Multidisciplinar Núcleo do Conhecimento, v. 1, p. 41 66 , 2019.

Disponível em:

https://www.nucleodoconhecimento.com.br/saude/caracterizacao-epidemiologica >.

OLIVEIRA, Elizarbio Carneiro de. Analise epidemiológica de acidentes de trabalho com exposição a material biológico entre profissionais de enfermagem, SANARE, Sobral, v14, n1, p27-32, 2015.

REIS, L.A.; GÓMEZ LA-ROTTA, E.I.; DINIZ, P.B.; AOKI, F.H.; JORGE, J. Occupational Exposure to Potentially Infectious Biological Material Among Physicians, Dentists, and Nurses at a University. Safety and Health at Work, v 10, n4, p 445-451. 2019.

RC: 78404

Disponível em: https://www.nucleodoconhecimento.com.br/gesundheit/biologischem$\underline{\text { material }}$ 
REIS, Phillipe Geraldo Teixeira de Abreu et al. Perfil epidemiológico de acidentes com material biológico entre estudantes de medicina em um pronto-socorro cirúrgico. Revista do Colégio Brasileiro de Cirurgiões. Rio de Janeiro: v. 40, n. 4, p.287-292, ago. 2013.

RUAS, Edna et al. Acidentes ocupacionais com materiais perfurocortantes em hospitais de Montes Claros-MG. Revista Mineira de Enfermagem, [S.I], v16, n3 p437-443, jul./set., 2012.

SANTOS Junior EP, et al. Acidentes de trabalho com material perfurocortante envolvendo profissionais e estudantes da área da saúde em hospital de referência. Rev. Bras. Med. Trab. [S.I], v 13, n 2, p 69-75, 2015

SANTOS Junior EP, et al. Acidentes de trabalho com material perfurocortante envolvendo profissionais e estudantes da área da saúde em hospital de referência. Rev. Bras. Med. Trab. [S.I], v 13, n 2, p 69-75, 2015

TALAAT, M. Occupational exposure to needlestick injuries and hepatitis B vaccination coverage among health care workers in Egypt. American Journal of Infection Control, v 31, n 8, p 469-474, 2003.

TAPIAS, L.F.; TAPIAS L.; TORRES, S.A. Accidentes biológicos en estudiantes de Medicina. Revista de la Universidad Industrial de Santander, [S.I], v 39, n 3, p 183-189, 2007.

VASCONCELOS, A. C. et al. Acidentes com materiais biológicos envolvendo estudantes da área da saúde no período de 2008-2018. In: SILVA, B. R. D. (Ed.). Difusão do conhecimento através das diferentes áreas da medicina 5. Ponta Grossa PR: Atena Editora, v.5, 2020. cap. 5, p.38-59.

Gesendet: März 2021.

$\mathrm{RC}: 78404$

Disponível em: https://www.nucleodoconhecimento.com.br/gesundheit/biologischem$\underline{\text { material }}$ 
Genehmigt: März 2021.

RC: 78404

Disponível em: https://www.nucleodoconhecimento.com.br/gesundheit/biologischem$\underline{\text { material }}$ 